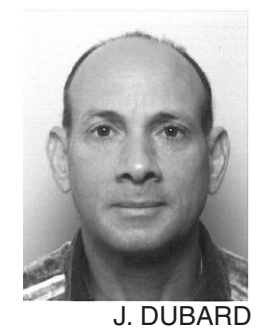

\title{
Spectroradiométrie UV : application au contrôle des cabines de bronzage
}

\section{UV spectroradiometry: Application to sunbeds control}

\author{
Jimmy DUBARD, Séverine GUIMIER, Thierry VALIN, Anne-Françoise OBATON et François BUTEAU \\ LNE-LCM (Laboratoire commun de métrologie LNE-Cnam), LNE (Laboratoire national de métrologie et d'essais), 29 avenue Roger Hennequin, \\ 78197 Trappes cedex, France, jimmy.dubard@lne.fr.
}

\section{Résumé}

Le contrôle des cabines de bronzage est obligatoire en France pour assurer la protection des consommateurs des dangers du rayonnement ultraviolet. Ce contrôle est réalisé par des organismes agréés à l'aide de spectroradiomètres à matrice de photodiodes qui doivent être étalonnés et traçables au SI. Ces spectroradiomètres présentent des défauts (sensibles à la lumière parasite) qu'il faut prendre en compte lors de leur étalonnage. Nous proposons une méthode simple d'étalonnage, par comparaison à un spectroradiomètre de référence, qui repose sur l'utilisation de sources dont la répartition spectrale est similaire à celle des sources placées dans les cabines de bronzage. Cet article présente le banc développé et la procédure mise en œuvre pour l'étalonnage des spectroradiomètres à matrice de photodiodes ainsi que l'évaluation des incertitudes d'étalonnage.

MOTS CLÉS : RAYONNEMENT, ULTRAVIOLET, SPECTRORADIOMÈTRE, SOURCE, ÉTALONNAGE, INCERTITUDE, CABINE DE BRONZAGE.

\begin{abstract}
Control of sunbeds is mandatory in France to ensure consumers protection against UV radiation. This control is performed by authorized body with array spectroradiometers that must be calibrated and traceable to SI. These spectroradiometers have defects (stray light) that need to be taken into account while performing the calibration. We propose a calibration method that is based on light sources which spectrum is similar to that of the light sources found in sunbeds, and by comparison to a reference spectroradiometer. This paper report on the bench and procedure developed to calibrate the array spectroradiometer as well as the calibration uncertainty.
\end{abstract}

KEY WORDS: RADIATION, ULTRAVIOLET, SPECTRORADIOMETER, SOURCE, CALIBRATION, UNCERTAINTY, SUNBED.

\section{Introduction}

Le rayonnement ultraviolet (UV) naturel ou artificiel est dangereux pour la vie, en particulier pour la peau et les yeux, car il peut occasionner des lésions cancéreuses [1-3]. En France, le mélanome de la peau est au $11^{\mathrm{e}}$ rang des cancers les plus fréquents. Par conséquent, l'utilisation d'un rayonnement UV artificiel tel que celui produit dans les cabines de bronzage doit être maîtrisé.

L'exploitation des cabines de bronzage est réglementée en France. Le Ministère de la Santé a mis en place des mesures pour cadrer l'utilisation des cabines de bronzage avec notamment le décret $n^{\circ}$ 2013-1261 du 27 décembre 2013 [4]. Les rampes et lampes de bronzage doivent être déclarées avant leur mise en service, être classées puis vérifiées par des organismes de contrôle agréés. La vérification par ces organismes agréés est réalisée à l'aide de spectroradiomètres UV à matrice de photodiodes qui doivent être étalonnés.

Les résultats (fig. 1) d'une comparaison réalisée en 2005 sur une grande partie du parc de spectroradiomètres UV à matrice de photodiodes utilisés en France par les organismes agréés ont montré la nécessité de mettre en place une procédure d'étalonnage spécifique afin de réduire la dispersion des mesures.

Cet article présente les caractéristiques du banc développé pour étalonner les spectroradiomètres UV à matrice 


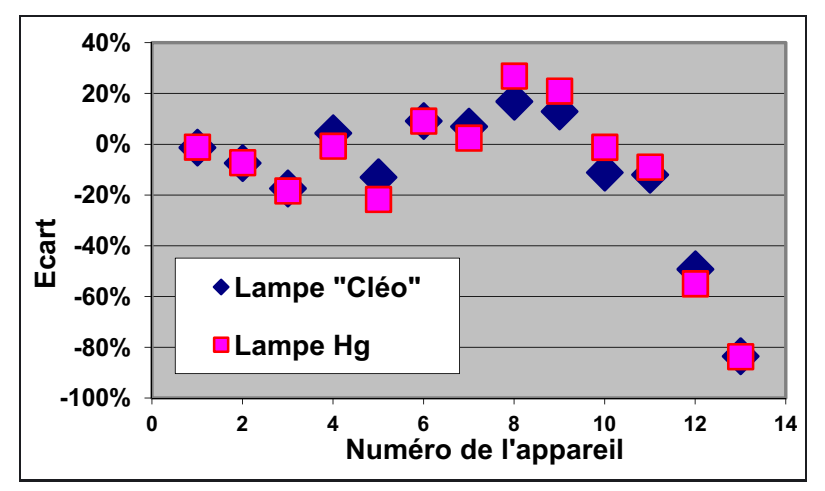

Fig. 1. - Résultats de la comparaison réalisée en 2005 sur des spectroradiomètres UV à matrice de photodiodes utilisés en France par les organismes agréés pour quantifier le rayonnement UVA.

de photodiodes, la procédure d'étalonnage et l'évaluation des incertitudes. Il décrit aussi la source UV étalon développée pour étalonner in situ un spectroradiomètre de référence en cas de caractérisation d'une cabine de bronzage sur site.

\section{Contexte}

L'arrêté du 9 décembre 1997 relatif aux conditions d'agrément d'organismes habilités à procéder au contrôle des installations de bronzage utilisant des rayonnements ultraviolets, donne des règles concernant le suivi métrologique des appareils de mesure :

- les instruments de mesure utilisés par ces sociétés de contrôle doivent être raccordés au moins une fois par an par le fabricant ou un organisme tiers;

- l'organisme de contrôle doit mettre en place une procédure d'auto-vérification à partir d'une source de référence selon une procédure précisée par écrit ;

- l'organisme de contrôle doit effectuer de façon régulière des mesures sur un banc de référence à la demande du ministère chargé de la santé.

En mars 2005, une campagne de mesures comparatives sur des spectroradiomètres UV à matrice de photodiodes utilisés en France par les organismes agréés a été organisée à l'initiative de la DGS (Direction Générale de la Santé). 13 appareils sur les 15 recensés en France, tous du même fabricant et de même type, ont fait l'objet de cette comparaison. Pour la comparaison, ils ont été placés face aux mêmes sources, une lampe à fluorescence de type «Cléo » et une lampe à vapeur de mercure haute pression. Les résultats de cette comparaison sont présentés sur la figure 1 où sont représentés, pour quantifier le rayonnement UVA, les écarts entre la valeur indiquée par chaque appareil et la valeur moyenne des mesures; les mesures des spectroradiomètres repérés « 12 » et «13» n'ont pas été prises en compte dans cette moyenne.

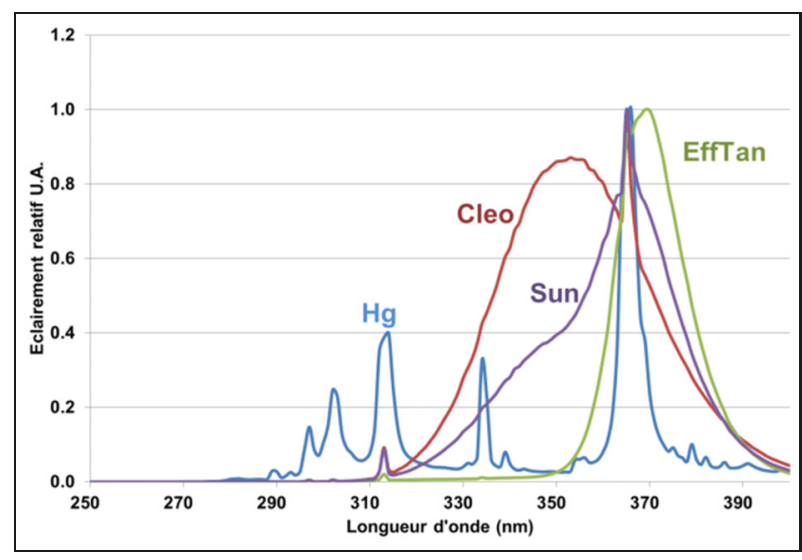

Fig. 2. - Répartitions spectrales des éclairements des lampes utilisées dans les cabines de bronzage.

\section{3. Étalonnage des spectroradiomètres UV}

L'ensemble des organismes habilités sont équipés de spectroradiomètres à matrice dont les principaux avantages sont :

- une compacité des appareils et une bonne robustesse qui les rendent facilement transportables;

- un temps d'acquisition rapide (quelque secondes) car le spectre est acquis en une seule fois avec l'utilisation de détecteur multicanal ;

- une alimentation simplifiée, par exemple par le port USB d'un ordinateur portable.

Mais ces spectroradiomètres à matrice présentent un fort taux de lumière parasite qui entraine des erreurs de mesure importantes dans le domaine spectral UV. Cette lumière parasite est due aux photons ayant des longueurs d'onde différentes de celle mesurée. Elle est due à la lumière polychromatique entrant dans le spectroradiomètre qui subit des réflections multiples à l'intérieur du spectroradiomètre et qui n'est pas distinguée des photons utiles par les détecteurs. Des techniques de corrections ont été développées sur la base d'une caractérisation de la LSF (Line Spread Function) à l'aide d'un laser accordable $[5,6]$. Mais cette méthode de caractérisation est coûteuse. Une autre méthode, plus simple, consiste à étalonner le spectroradiomètre à matrice par comparaison à un spectroradiomètre de référence en utilisant des sources spécifiques similaires à celles rencontrées dans les cabines de bronzage. C'est cette méthode qui a été mise en œuvre au LNE.

\subsection{Sources de lumière UV}

Les lampes équipant les cabines de bronzage sont de plusieurs types selon les différents fournisseurs du marché. La mesure de la répartition spectrale de l'émission de ces lampes a permis de créer quatre familles de lampes selon le spectre d'émission. Elles sont repérées : « $\mathrm{Hg} »$, «Cléo », «Sun» et «EffTan». Les répartitions spectrales de chaque type de lampes sont présentées sur la figure 2. 


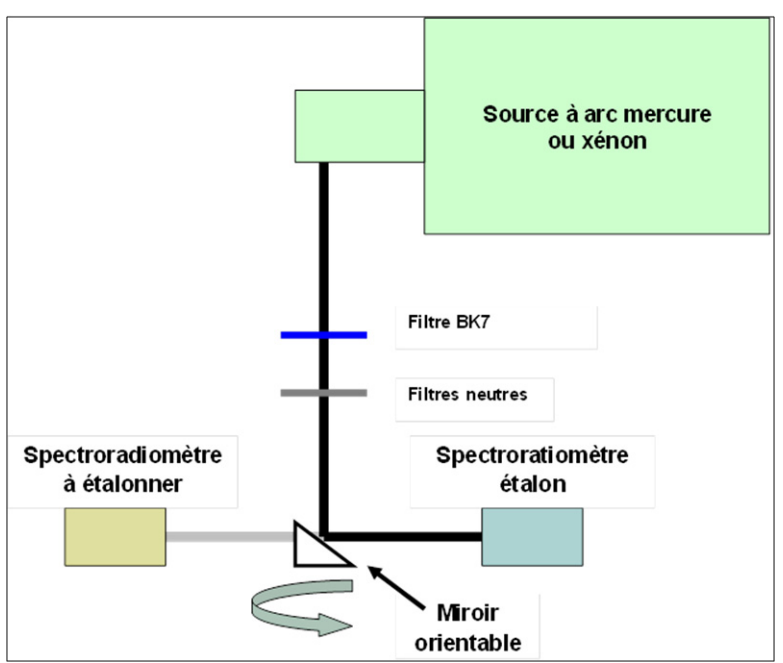

Fig. 3. - Banc d'étalonnage des spectroradiomètres UV à matrice de photodiodes fonctionnant avec un simulateur solaire.

Elle montre que ces lampes émettent principalement dans le domaine spectral de l'UVA (315 nm-380 nm).

\subsection{Bancs d'étalonnage des spectroradiomètres}

Deux bancs d'étalonnage ont été mis en œuvre pour tenir compte des différentes géométries des sources disponibles.

\subsubsection{Banc « Source collimatée »}

Le principe du banc « Source collimatée » est illustré sur la figure 3. Il comprend un simulateur solaire dont le faisceau lumineux est mis en forme avec un jeu de lentilles pour réaliser un faisceau collimaté.

Le simulateur solaire est équipé d'une lampe à arc à vapeur de mercure haute pression $(\mathrm{HgHP})$ ou d'une lampe au xénon. Un filtre de type BK7 est interposé sur le faisceau de manière à éliminer les longueurs d'onde inférieures à $280 \mathrm{~nm}$. Des filtres neutres sont utilisés pour réduire l'éclairement et ne pas saturer les appareils de mesure. Le faisceau dont le diamètre est de $30 \mathrm{~mm}$ est ensuite dirigé vers le spectroradiomètre étalon ou le spectroradiomètre à étalonner à l'aide d'un miroir orientable.

\subsubsection{Banc « Source étendue »}

Le banc «Source étendue » est constitué d'un luminaire à tubes fluorescents. À cause de son encombrement, nous avons disposé le luminaire verticalement face à une table optique à une distance des lampes de $25 \mathrm{~cm}$ (distance de mesure spécifiée dans le décret $\mathrm{N}^{\circ} 2013-1261 \mathrm{du}$ 27 décembre 2013). Les sondes du spectroradiomètre de référence et des spectroradiomètres à étalonner sont placées en bout de la table optique face aux lampes (fig. 4). Le faisceau vu par les spectroradiomètres est donc multidirectionnel. Pour minimiser les erreurs de mesure dues aux conditions géométriques d'éclairement, les sondes sont placées l'une au-dessus de l'autre.

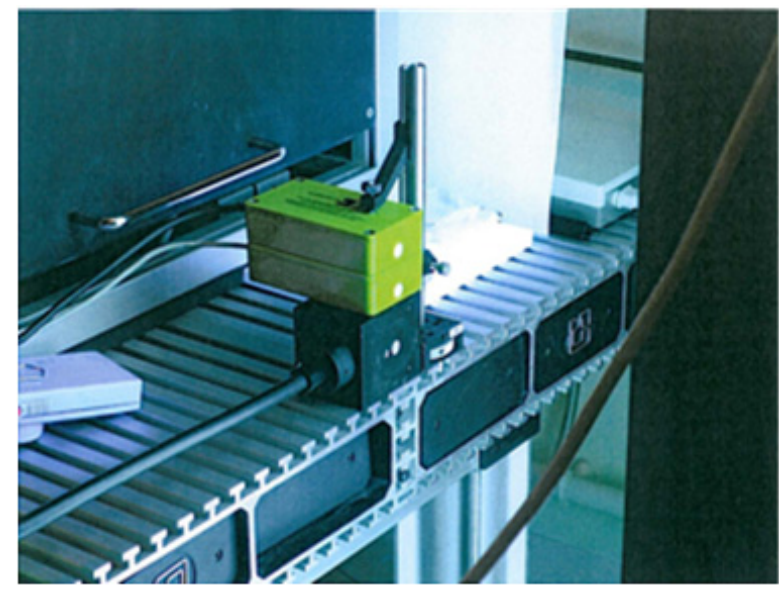

Fig. 4. - Banc d'étalonnage des spectroradiomètres UV à matrice de photodiodes fonctionnant avec un luminaire à tubes fluorescents.

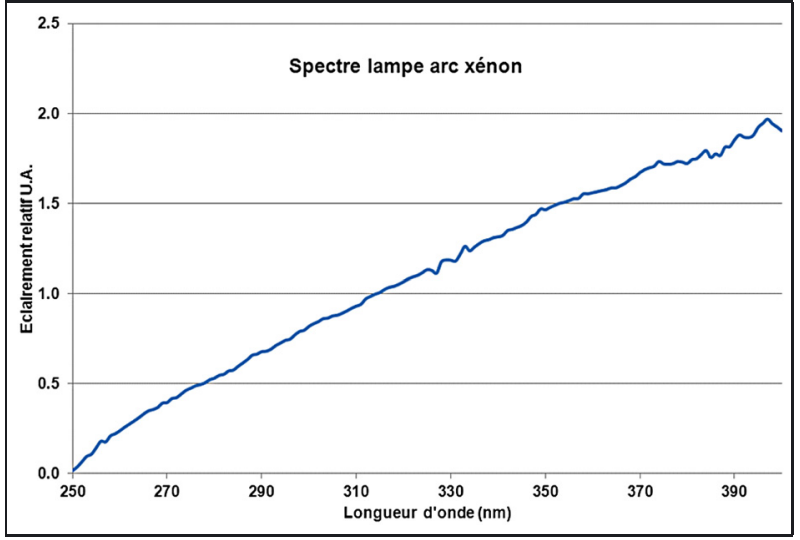

Fig. 5. - Répartition spectrale de l'éclairement de la lampe à arc xénon utilisée pour le contrôle du fonctionnement des spectroradiomètres des organismes agréés.

\subsection{Spectroradiomètre de référence}

Le spectroradiomètre de référence, de marque OPTRONIC LABORATORIES et de type OL754, est conçu sur la base d'un monochromateur double associé à un détecteur de type photomultiplicateur. La sonde est une sphère intégrante reliée au monochromateur par un faisceau de fibres optiques.

Le domaine spectral de sensibilité du spectroradiomètre de référence est $200 \mathrm{~nm}-800 \mathrm{~nm}$. La largeur spectrale d'analyse est variable et est choisie à $1 \mathrm{~nm}$ pour l'étalonnage des spectroradiomètres.

\subsection{Procédure d'étalonnage des spectroradiomètres UV}

La procédure d'étalonnage comprend quatre étapes.

\subsection{1. Étape 1-Vérification du bon fonctionnement du spectroradiomètre}

Le simulateur solaire du banc «Source collimatée » est constitué d'une lampe à arc xénon qui émet sur un domaine spectral étendu de l'UV à l'infrarouge (fig. 5). 
Cette large étendue spectrale permet de vérifier la capacité du spectroradiomètre à détecter le rayonnement sur l'ensemble du domaine spectral requis $(250 \mathrm{~nm}-450 \mathrm{~nm})$.

\subsection{2. Étape 2-Vérification de l'échelle des longueurs d'onde du spectroradiomètre à étalonner}

La caractérisation en longueur d'onde du spectroradiomètre sur le domaine spectral $250 \mathrm{~nm}-400 \mathrm{~nm}$ est effectuée en analysant les résultats d'une mesure du spectre d'émission de la lampe à vapeur de mercure dont les raies d'émission sont connues (fig. 2) [7]. On relève la longueur d'onde «pic » de chacune des raies mesurées et on la compare à la longueur d'onde de référence. Les longueurs d'onde de référence sélectionnées sont $297 \mathrm{~nm}, 313 \mathrm{~nm}$ et $365 \mathrm{~nm}$. Cette caractérisation permet aussi d'évaluer la largeur de bande spectrale d'analyse du spectroradiomètre.

\subsection{3. Étape 3-Étalonnage en éclairement énergétique spectrique}

La méthode utilisée est une méthode par comparaison au spectroradiomètre de référence, ceci pour différentes configurations spectrales de sources correspondant aux deux bancs mis en œuvre (lampe HgHP, tubes fluorescents). L'étalonnage en éclairement énergétique spectrique est effectué sur le domaine spectral $250 \mathrm{~nm}-$ $400 \mathrm{~nm}$, en ajustant le pas du spectroradiomètre de référence à $1 \mathrm{~nm}$; le pas du spectroradiomètre à étalonner étant fixe et inférieur ou égal à $1 \mathrm{~nm}$.

\subsection{4. Étape 4-Détermination des facteurs correctifs}

Les mesures effectuées à l'étape 3 permettent de caractériser quantitativement le spectroradiomètre par la détermination de facteurs correctifs pour les domaines spectraux dUVA et dUVB. Les domaines dUVA et dUVB (« $\mathrm{d} »$ pour dermatologie) diffèrent des domaines UVA et UVB (définis par la CIE [8]) par la longueur d'onde de séparation des domaines qui est de $320 \mathrm{~nm}$ entre dUVA et dUVB, et $315 \mathrm{~nm}$ entre UVA et UVB.

Ces facteurs correctifs sont déterminés de la façon suivante pour chaque source repérée $« k »$ :

- à partir des éclairements énergétique spectriques mesurés $E_{k, l}$ par le spectroradiomètre de référence $(l=\mathrm{S})$ et par le spectroradiomètre à caractériser ( $l=$ UDC), on calcule les éclairements érythémals $E_{\text {er-dUVA }}(\mathrm{k}, \mathrm{l})$ et $E_{\text {er-dUVB }}(k, l)$

$$
E_{\text {er-dUVA }}(k, l)=\int_{320}^{400} E_{k, l}(\lambda) S_{\text {er }}(\lambda) d \lambda
$$

et

$$
E_{\text {er-dUVB }}(k, l)=\int_{250}^{320} E_{k, l}(\lambda) S_{\text {er }}(\lambda) d \lambda
$$

où $S_{\text {er }}(\lambda)$ est la courbe d'action de l'érythème $[9,10]$.
- Les facteurs correctifs $F A_{k}$ et $F B_{k}$ sont obtenus par les équations suivantes :

$$
F A_{k}=\frac{E_{\mathrm{er}-\mathrm{dUVA}}(k, \mathrm{~S})}{E_{\mathrm{er}-\mathrm{dUVA}}(k, \mathrm{UDC})} \text { et } F B_{k}=\frac{E_{\mathrm{er}-\mathrm{dUVB}}(k, \mathrm{~S})}{E_{\mathrm{er}-\mathrm{dUVB}}(k, \mathrm{UDC})}
$$

\section{Incertitude d'étalonnage de spectroradiomètres UV}

Les spectroradiomètres sont étalonnés par comparaison au spectroradiomètre de référence du laboratoire lui-même étalonné avant chaque campagne d'étalonnage. Les sources d'incertitude d'étalonnage sont les suivantes :

- répétabilité des mesures;

- étalonnage du spectroradiomètre de référence;

- distance entre le spectroradiomètre et les sources ;

- homogénéité du faisceau d'éclairement;

- température ambiante autour du banc d'étalonnage.

\subsection{Répétabilité des mesures}

Pour chaque type de source, les mesures sont effectuées trois fois. L'écart type relatif des mesures varie en fonction de la longueur d'onde de mesure. Il est de $10 \%$ pour les longueurs d'onde inférieures à $310 \mathrm{~nm}, 4 \%$ pour les longueurs d'onde entre $310 \mathrm{~nm}$ et $320 \mathrm{~nm}$ et $2,5 \%$ pour les longueurs d'onde supérieures à $320 \mathrm{~nm}$.

\section{2. Étalonnage du spectroradiomètre de référence}

Le spectroradiomètre de référence est étalonné en utilisant une lampe au deutérium pour l'étalonnage sur le domaine spectral $250 \mathrm{~nm}-300 \mathrm{~nm}$, et une lampe tungstène-halogène de type FEL sur le domaine spectral 290 nm-450 nm. L'incertitude d'étalonnage est évaluée à partir des contributions suivantes :

\section{- Répétabilité des mesures}

Cette incertitude est évaluée en effectuant 5 mesures successives sur une source stable (lampes deutérium et tungstène-halogène). Pour les longueurs d'onde inférieures à $300 \mathrm{~nm}$, la répétabilité est de $1 \%$. Pour les longueurs d'onde entre $300 \mathrm{~nm}$ et $320 \mathrm{~nm}$, elle est de $0,5 \%$. Pour les longueurs d'onde supérieures à $320 \mathrm{~nm}$, elle est de $0,25 \%$.

\section{- Lampes étalons d'éclairement spectrique}

L'incertitude d'étalonnage des lampes est fournie par le certificat d'étalonnage des lampes étalons utilisées pour l'étalonnage du spectroradiomètre. Elle est de $1 \%$ pour les longueurs d'onde situées entre $250 \mathrm{~nm}$ et $299 \mathrm{~nm}$, de $0,8 \%$ de $300 \mathrm{~nm}$ à $349 \mathrm{~nm}$ et $0,7 \%$ pour des longueurs d'onde $\geq 350 \mathrm{~nm}$.

\section{- Dérive des lampes étalons}

Elle est évaluée à partir des valeurs des éclairements spectriques obtenues lors des différents étalonnages des lampes étalons réalisés au cours du temps. 
Tableau 1

Bilan d'incertitude d'étalonnage du spectroradiomètre de référence.

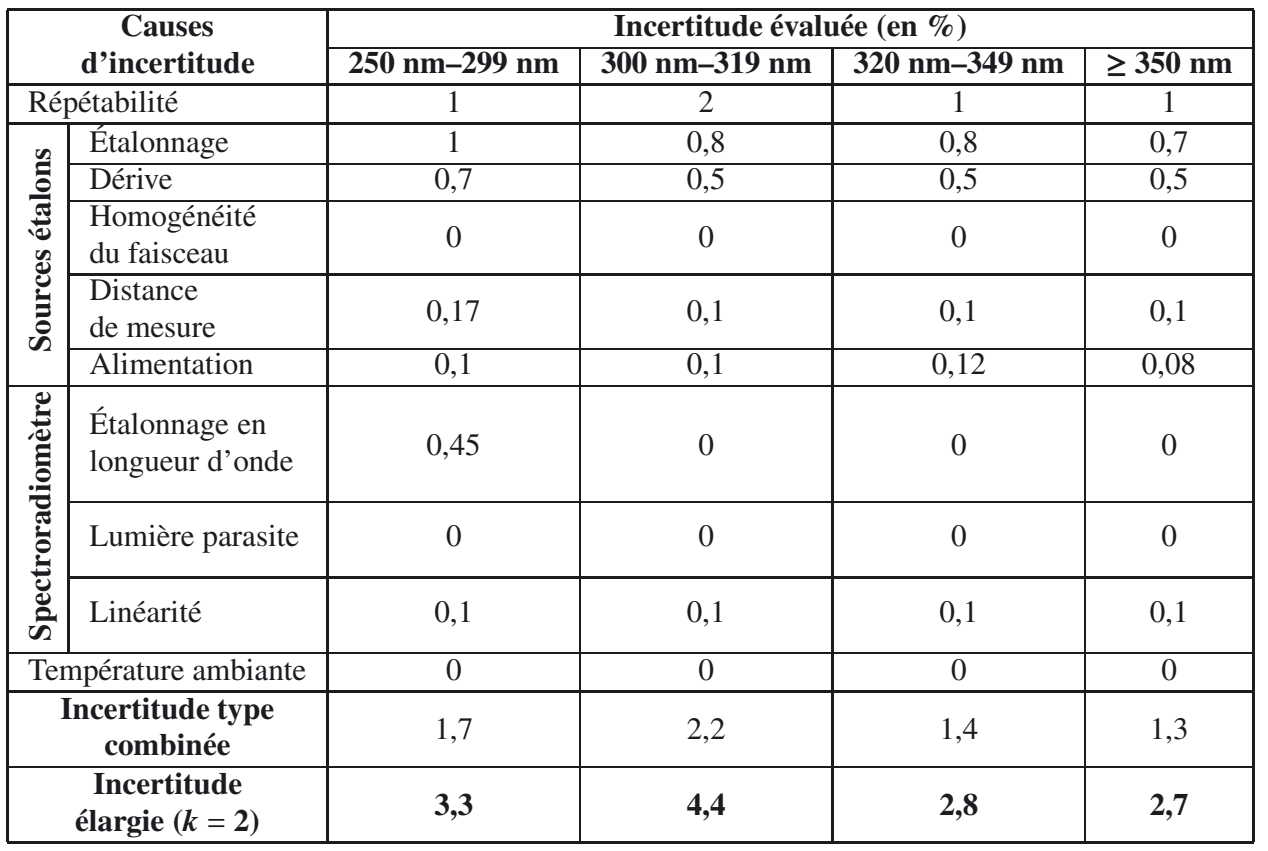

\section{- Courant d'alimentation des lampes étalon}

Pour la lampe au deutérium, le courant d'alimentation est ajusté avec une incertitude de $0,4 \mathrm{~mA}$ pour un courant d'alimentation de $0,3 \mathrm{~A}$. L'incertitude relative sur l'éclairement produit est de $0,1 \%$ sur le domaine spectral $250 \mathrm{~nm}-320 \mathrm{~nm}$.

Pour la lampe de type FEL, le courant d'alimentation est mesuré avec une incertitude relative de $0,025 \%$. Ceci engendre une incertitude relative de $0,12 \%$ à $320 \mathrm{~nm}$ et de $0,08 \%$ à $400 \mathrm{~nm}$ sur l'étalonnage du spectroradiomètre.

\section{- Distance de mesure}

La lampe étalon de type FEL est positionnée à une distance de $500 \mathrm{~mm}$ mesurée avec une incertitude de $0,25 \mathrm{~mm}$. L'incertitude relative sur la mesure de l'éclairement produit est de 0,1\%. La lampe étalon au deutérium est utilisée à une distance du spectroradiomètre de $300 \mathrm{~mm}$ mesurée avec une incertitude de $0,25 \mathrm{~mm}$. L'incertitude relative induite sur la mesure de l'éclairement produit est donc de 0,17\%.

\section{- Homogénéité du faisceau}

Cette incertitude est évaluée en déplaçant la sonde du spectroradiomètre dans un plan perpendiculaire au faisceau produit par la lampe étalon autour du point de mesure de référence. Un déplacement de $1 \mathrm{~cm}$ par rapport au point de mesure de référence montre que les variations sure l'éclairement spectrique mesuré sont équivalentes à la répétabilité des mesures. Par conséquent, l'incertitude due à l'homogénéité du faisceau est négligeable.

\section{- Étalonnage en longueur d'onde du spectroradiomètre}

Le spectroradiomètre est calibré en longueur d'onde avec une incertitude de $0,05 \mathrm{~nm}$. Cette incertitude entraîne une incertitude sur la mesure de l'éclairement qui atteint $1 \%$ pour des longueurs d'onde entre $300 \mathrm{~nm}$ et $320 \mathrm{~nm}$.

\section{- Influence de la largeur spectrale d'analyse du spectroradiomètre}

La largeur spectrale d'analyse est de 1,2 nm. La variation de l'éclairement sur cet intervalle spectral entraîne un décalage effectif de la longueur d'onde centrale de la lumière analysée par le spectroradiomètre. Ce décalage peut atteindre $0,07 \mathrm{~nm}$ sur le domaine spectral $300 \mathrm{~nm}-$ $320 \mathrm{~nm}$ ce qui génère une incertitude sur la mesure de l'éclairement de 1,4\%.

\section{- Lumière parasite}

Le niveau de lumière parasite est évalué en mesurant l'éclairement spectrique produit par une source blanche, sans et avec des filtres passe-haut de type WG320 ou WG340. Les résultats indiquent que la lumière parasite est négligeable, inférieure à $10^{-3}$.

\section{- Température}

Dans les conditions du laboratoire avec une température de $23{ }^{\circ} \mathrm{C}$ contrôlée à $\pm 2{ }^{\circ} \mathrm{C}$, l'influence de la température est négligeable, et est inclus dans la répétabilité des mesures.

Le bilan d'incertitude d'étalonnage du spectroradiomètre de référence est indiqué dans le tableau 1. 
Tableau 2

Bilan d'incertitude d'étalonnage des spectroradiomètres.

\begin{tabular}{|l|c|c|c|c|}
\hline \multirow{2}{*}{$\begin{array}{c}\text { Causes } \\
\text { d'incertitude }\end{array}$} & \multicolumn{4}{|c|}{ Incertitude évaluée (en \%) } \\
\cline { 2 - 5 } & $\mathbf{2 5 0} \mathbf{~ n m - 3 0 9} \mathbf{~ m m}$ & $\mathbf{3 1 0} \mathbf{~ n m - 3 1 9} \mathbf{~ n m}$ & $\mathbf{3 2 0} \mathbf{~ n m - 3 4 9} \mathbf{~ n m}$ & $\mathbf{3 5 0} \mathbf{~ n m - 4 0 0 ~} \mathbf{~ m}$ \\
\hline Répétabilité & 10 & 4 & 1,4 & 2,5 \\
\hline $\begin{array}{l}\text { Étalonnage du } \\
\text { spectroradiomètre }\end{array}$ & 1,7 & 2,2 & 0,6 & 1,3 \\
\hline $\begin{array}{c}\text { Distance de mesure } \\
\text { par rapport aux sources }\end{array}$ & 0,6 & 0,6 & 2 & 0,6 \\
\hline Homogénéité du faisceau & 2 & 2 & 0 & 2 \\
\hline Température ambiante & 0 & 0 & 3,5 & 3,5 \\
\hline $\begin{array}{c}\text { Incertitude type } \\
\text { combinée }\end{array}$ & 10 & 5,0 & $\mathbf{7 , 1}$ & $\mathbf{7 , 0}$ \\
\hline $\begin{array}{c}\text { Incertitude } \\
\text { élargie }(\boldsymbol{k}=\mathbf{2})\end{array}$ & $\mathbf{2 1}$ & $\mathbf{1 0}$ & & \\
\hline
\end{tabular}

\subsection{Distance spectroradiomètre-source}

Sur le banc « simulateur solaire », le faisceau est collimaté. L'incertitude d'étalonnage due à la distance de mesure est négligeable.

Pour le banc "luminaire à tubes », les sondes du spectroradiomètre de référence et du spectroradiomètre à étalonner sont placées à égale distance de la source (250 mm) déterminée avec une incertitude de $2 \mathrm{~mm}$. La variation du signal mesuré en changeant la distance par rapport à la source de $2 \mathrm{~mm}$ est de $1 \%$ (distribution rectangulaire). L'incertitude associée sur l'étalonnage ou la mesure de l'éclairement est de 0,6\%.

\subsection{Homogénéité du faisceau}

Le diamètre de la surface sensible des sondes du spectroradiomètre de référence et du spectroradiomètre à étalonner est de $1 \mathrm{~cm}$.

Sur le banc « simulateur solaire» dont le faisceau collimaté a un diamètre de $5 \mathrm{~cm}$, les sondes sont alignées avec un laser. L'incertitude due à l'homogénéité du faisceau est estimée en déplaçant la sonde dans la section du faisceau. Elle est de $2 \%$.

Sur le banc « luminaire à tubes » la source est étendue. Les sondes du spectroradiomètre de référence et du spectroradiomètre à étalonner sont placées l'une au-dessus de l'autre et les surfaces sensibles sont espacées de $5 \mathrm{~cm}$ à $7 \mathrm{~cm}$. Une étude de la variation du signal dans un plan parallèle à la source a montré que l'incertitude due à l'homogénéité est de $2 \%$.

\subsection{Température ambiante}

La salle où est réalisé l'étalonnage des spectroradiomètres pour les cabines de bronzage est régulée en température à $23{ }^{\circ} \mathrm{C} \pm 2{ }^{\circ} \mathrm{C}$. Le spectroradiomètre de référence est étalonné dans un milieu ambiant similaire et la variation de la température sur le domaine $21^{\circ} \mathrm{C}-25^{\circ} \mathrm{C}$ n'a pas d'effet notable sur cet étalonnage. Par conséquent, l'incertitude due à la température ambiante est négligeable.
L'incertitude donnée dans le tableau 2 concerne la mesure de la répartition spectrale d'émission des sources utilisées pour l'étalonnage du spectroradiomètre. Pour l'incertitude sur les facteurs de correction sur les domaines dUVA et dUVB, il faut prendre en compte la courbe d'action de l'érythème.

Pour l'incertitude sur le facteur $F B$, il faut considérer le domaine spectral $310 \mathrm{~nm}-319 \mathrm{~nm}$ car les valeurs de l'éclairement spectrique pondéré par la courbe d'action de l'érythème sont négligeables pour les longueurs d'onde inférieures à $310 \mathrm{~nm}$.

Pour l'incertitude sur le facteur $F A$, il faut considérer le domaine spectral $320 \mathrm{~nm}-349 \mathrm{~nm}$ car les valeurs de l'éclairement spectrique pondéré par la courbe d'action de l'érythème contribuent plus faiblement pour les longueurs d'onde supérieures à $350 \mathrm{~nm}$.

L'incertitude d'étalonnage $(k=2)$ pour les facteurs de correction est de $7,1 \%$ pour $F A$ et $10 \%$ pour $F B$.

Pour les étalonnages courants, compte tenu des faibles éclairements produits par les sources dans le domaine UVB, l'incertitude relative du facteur $F B$ est majorée et est de $15 \%$.

\section{Caractérisation des cabines de bronzage sur site}

En cas de contestation des mesures réalisées sur une cabine de bronzage avec un spectroradiomètre à matrice de photodiodes, une validation à l'aide d'un spectroradiomètre de référence à balayage avec un monochromateur double doit être effectuée sur site. Les prestations sur site sont complexes à mettre en œuvre : accessibilité à la source, environnement non-contrôlé (température, perturbation électromagnétique, vibration, rayonnement lumineux...). Le spectroradiomètre de référence est sensible aux paramètres environnementaux et il est nécessaire de vérifier le calibrage in situ.

Une source de contrôle transportable a été développée pour réaliser cette vérification et sera utilisée dans un environnement non maîtrisé. Par conséquent, dans son utilisation, l'espace entre la lampe et la sonde 


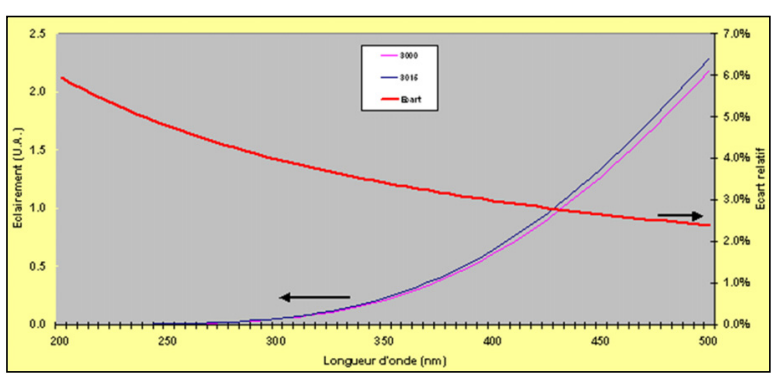

Fig. 6. - Variation de l'éclairement énergétique spectrique de la lampe avec le courant d'alimentation (température de couleur).

du spectroradiomètre de référence doit être isolé de tous les rayonnements parasites provenant des sources de lumière présentes dans la salle où est réalisée la mesure.

\subsection{Cahier des charges de la source transportable de contrôle}

La source doit fournir un éclairement spectrique absolu (exprimé en $\mathrm{W} \cdot\left(\mathrm{m}^{2} \cdot \mathrm{nm}\right)^{-1}$ ) de manière à pouvoir déterminer un facteur correctif à appliquer aux mesures réalisées sur site en cas de variation de la réponse du spectroradiomètre de référence entre son étalonnage réalisé en laboratoire et sa vérification sur site.

La source doit émettre un spectre continu. La puissance consommée et les caractéristiques électriques doivent être compatibles avec celles d'une alimentation stabilisée transportable.

L'alimentation électrique doit avoir une bonne stabilité en courant de manière à ce qu'elle n'apporte pas une contribution trop importante à l'incertitude d'étalonnage. La lampe tungstène a une température de couleur de fonctionnement de l'ordre de $3000 \mathrm{~K}$. Une variation relative de $1 \%$ de son courant d'alimentation entraîne une variation de la température de couleur de $15 \mathrm{~K}$. La figure 6 montre la distribution spectrale relative du corps noir à $3000 \mathrm{~K}$ et à $3015 \mathrm{~K}$ sur le domaine spectral $200 \mathrm{~nm}-500 \mathrm{~nm}$ ainsi que l'écart relatif entre ces deux répartitions spectrales. Cet écart varie entre 4,8\% à $250 \mathrm{~nm}$ et 3,0\% à $400 \mathrm{~nm}$. Pour avoir une composante d'incertitude sur le spectre d'émission de la source due à la stabilité de la source de $1 \%(k=2)$ alors il faut que le courant d'alimentation de la source soit stable à mieux que $0,2 \%$.

Le cahier des charges de la source transportable est donc constitué des points suivants :

- l'ensemble comprenant la lampe et la sonde du spectroradiomètre doit être isolé de l'extérieur par une structure mécanique;

- le rayonnement émis par la source doit être à spectre continu ;

- l'alimentation électrique de la lampe doit être stable à mieux que $0,2 \%$.

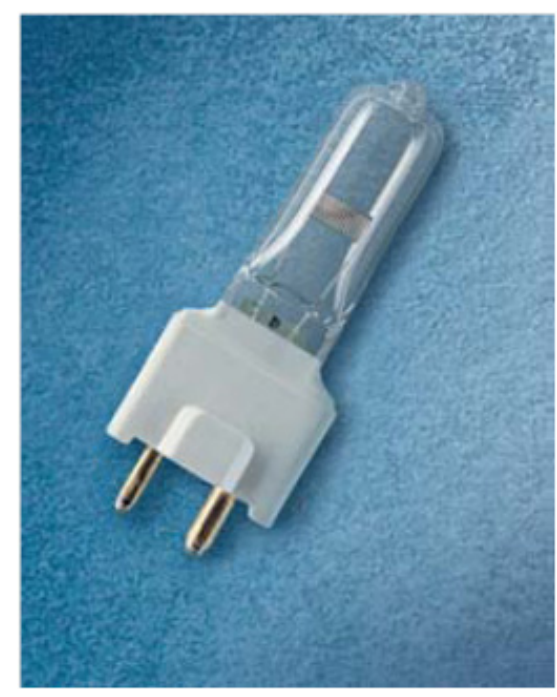

Fig. 7. - Lampe tungstène-halogène de la source transportable de contrôle.

\subsection{Alimentation stabilisée}

Le laboratoire a depuis plusieurs années acquis des alimentations électriques de marque Agilent et de la série E36XXA. Le modèle E3634A présente les caractéristiques suivantes :

- il est compact, transportable et programmable;

- l'alimentation est progressive et peut être gérée soit en tension, soit en courant. Ceci permet de préserver la lampe et de lui garantir une durée de vie optimale;

- il intégre un voltmètre et un ampèremètre, avec un affichage à 4 digits, qui peuvent être étalonnés ;

- la puissance maximale délivrée est $200 \mathrm{~W}$ en régime continu, selon deux configurations tension/courant (tension 0-25 V et courant 0-7 A ou tension 0-50 V et courant 0-4 A), qui peut donc être optimisée en fonction de la lampe choisie ;

- la stabilité du courant $I$ spécifiée par le constructeur est : $0,1 \% I+1 \mathrm{~mA}$.

\subsection{Lampe}

Pour l'étalonnage sur site du spectroradiomètre, nous devons disposer d'une source à spectre large pour couvrir le domaine spectral de $250 \mathrm{~nm}$ à $800 \mathrm{~nm}$. Une lampe tungstène-halogène répond à ce critère. La température de couleur, de l'ordre de $3000 \mathrm{~K}$ à $3200 \mathrm{~K}$, permet en effet d'obtenir une contribution significative du rayonnement dans le domaine spectral UV de $250 \mathrm{~nm}$ à $300 \mathrm{~nm}$.

La lampe sélectionnée est de marque OSRAM de type 64643 (fig. 7). Elle présente les paramètres suivants :

- elle est compacte (hauteur inférieure à $50 \mathrm{~mm}$ ) ;

- ses caractéristiques électriques sont optimisées par rapport à l'alimentation disponible : puissance de $150 \mathrm{~W}$ sous $24 \mathrm{~V}$ soit un courant nominal de 6,25 A; combinée à cette alimentation, la stabilité en courant est de $7 \mathrm{~mA}$, soit environ $0,1 \%$ en valeur relative; 
Tableau 3

Caractéristiques de la lampe tungstène-halogène de la source transportable de contrôle.

\begin{tabular}{|l|c|}
\hline Marque & OSRAM \\
\hline Type & 64643 \\
\hline Puissance (W) & 150 \\
\hline Tension (V) & 24 \\
\hline Durée de vie (heures) & 100 \\
\hline Position de fonctionnement & Culot en bas \\
\hline Type de culot & GY9.5 \\
\hline
\end{tabular}

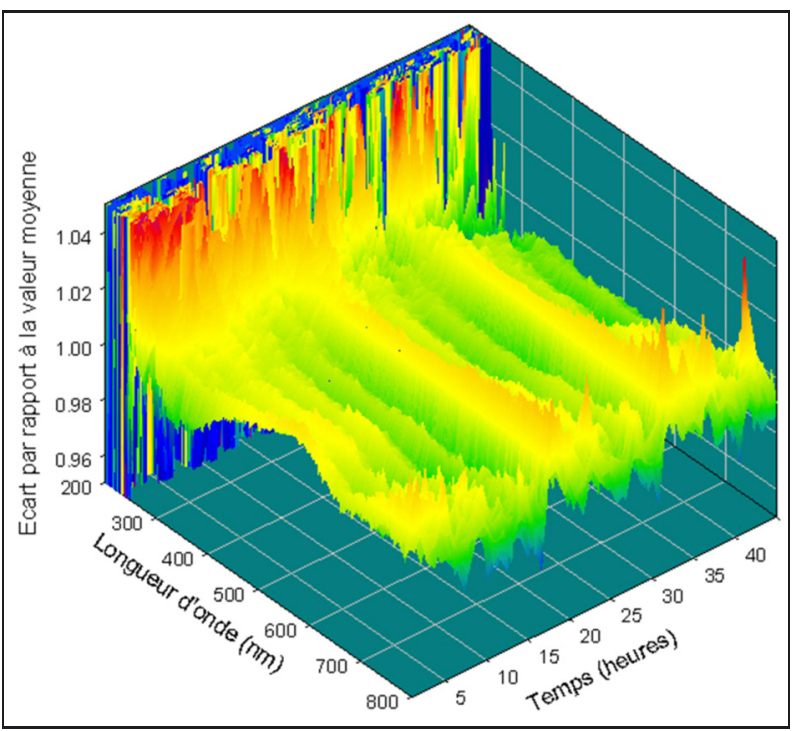

Fig. 8. - Caractérisation de la stabilité de la lampe tungstènehalogène de la source transportable de contrôle.

- elle a une durée de vie supérieure à $100 \mathrm{~h}$;

- son culot est de type GY9.5 lui conférant une stabilité mécanique optimale à l'intérieur de la source transportable.

Les caractéristiques de la lampe sont résumées dans le tableau 3.

L'étude de la stabilité du rayonnement est réalisée en mesurant le l'éclairement spectrique de la lumière émise en utilisant un spectroradiomètre de type OL756. Le domaine spectral de mesure s'étend de $200 \mathrm{~nm}$ à $800 \mathrm{~nm}$ avec un pas de $1 \mathrm{~nm}$. La lampe est montée dans la source transportable. Les mesures sont effectuées sur un temps de fonctionnement de $45 \mathrm{~h}$.

La stabilité de la lampe (fig. 8) est caractérisée par la représentation des écarts par rapport à la moyenne des éclairements spectriques mesurés en fonction de la longueur d'onde et du temps de fonctionnement exprimé en heure.

Les oscillations en fonction du temps de fonctionnement, correspondent à une variation de l'éclarement spectrique au cours de la journée due à l'évolution de la température des différents éléments utilisés : spectroradiomètre, alimentation stabilisée. Cette variation est de l'ordre de $2 \%$ à $300 \mathrm{~nm}$ et $1 \%$ à $400 \mathrm{~nm}$.
Cette étude apporte des informations sur le fonctionnement du spectroradiomètre dans des conditions d'utilisation sur site et permet de déterminer la contribution de la température de l'appareil à l'incertitude de mesure.

La figure 9 montre un vieillissement de la lampe de l'ordre de $1 \%$ sur une durée de fonctionnement de $45 \mathrm{~h}$. Une périodicité d'étalonnage de la source de $20 \mathrm{~h}$ de fonctionnement est préconisée. Ceci permet de réaliser 40 opérations de contrôle du spectroradiomètre sur site (15 min de stabilisation en température et $15 \mathrm{~min}$ de mesure).

\subsection{Réalisation de la source transportable de contrôle}

La source transportable est constituée de trois parties (fig. 10 et 11) : support de lampe, support de la sonde du spectroradiomètre et tube de liaison.

La distance entre la lampe et l'entrée de la sonde est de $210 \mathrm{~mm}$. L'ensemble des pièces sont anodisées. Le tube de liaison comporte des stries pour réduire les réflexions parasites. La lampe est soumise à un refroidissement naturel par convexion au travers d'ouverture sur le boîtier.

La source transportable (fig. 11) a une masse de moins de $3 \mathrm{~kg}$. Elle est mise dans un conteneur (de dimensions $700 \mathrm{~mm} \times 500 \mathrm{~mm} \times 500 \mathrm{~mm}$ ) avec l'alimentation électrique et des accessoires pour l'utilisation sur site. La source est utilisée sur le domaine spectral de $230 \mathrm{~nm}$ à $800 \mathrm{~nm}$ et utilisable dans l'infrarouge jusqu'à $2500 \mathrm{~nm}$ pour l'étalonnage de spectroradiomètres à spectre large. La figure 12 montre la comparaison entre l'éclairement spectrique mesuré avec le spectroradiomètre OL754, en laboratoire et sur site, pour la lampe H1.

\section{5. Évaluation des incertitudes de mesure sur site}

Trois sources d'incertitude prépondérantes sont identifiées pour l'évaluation de l'incertitude de mesure de l'éclairement énergétique spectrique produit par la source transportable : la répétabilité, l'étalonnage du spectroradiomètre et le courant d'alimentation de la lampe.

\section{- Incertitude de répétabilité}

Cette incertitude est évaluée en calculant l'écart type relatif de 10 mesures de l'éclairement spectrique de la source transportable, entre $250 \mathrm{~nm}$ et $400 \mathrm{~nm}$.

\section{- Incertitude sur l'étalonnage du spectroradiomètre}

Cette incertitude a déjà été présentée en paragraphe 4 et le bilan est synthétisé dans le tableau 2.

\section{- Incertitude due au courant d'alimentation de la lampe}

Le courant d'alimentation de la lampe est ajusté avec une incertitude de $1 \mathrm{~mA}$ pour un courant de $6,25 \mathrm{~A}$, ce qui conduit à une incertitude en valeur relative de $0,02 \%$. La contribution sur l'incertitude de l'éclairement produit 


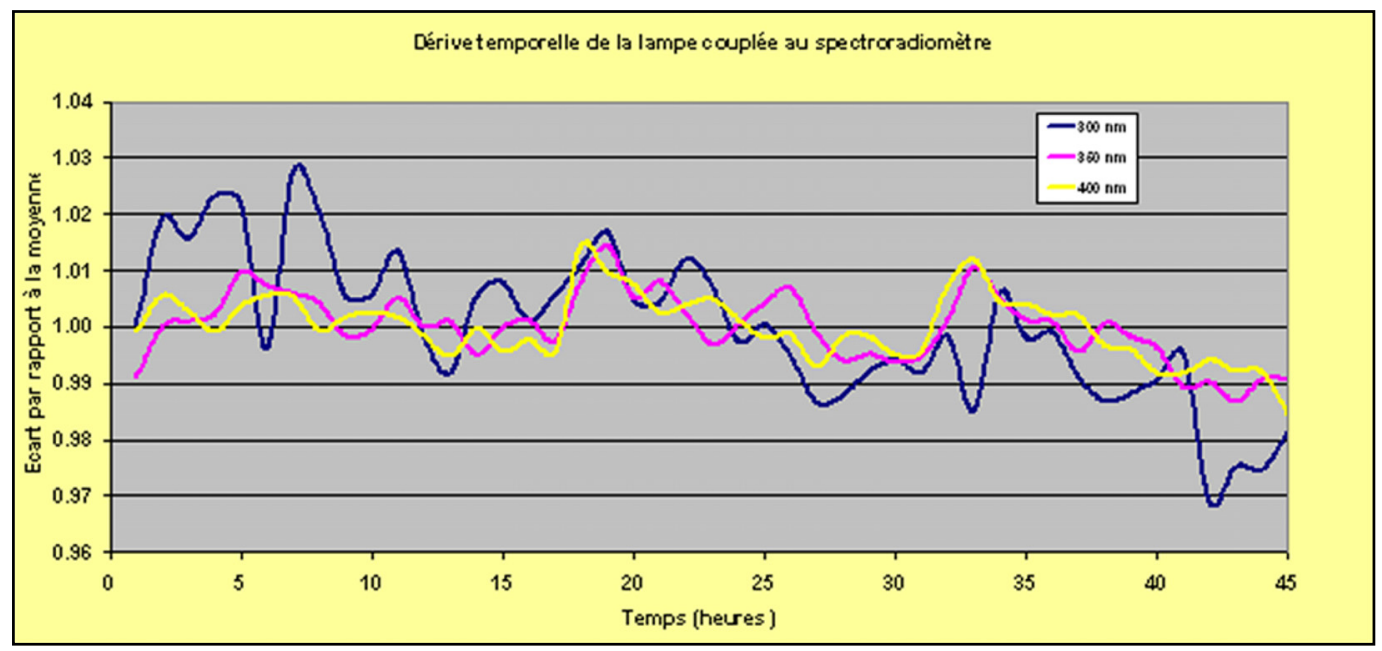

Fig. 9. - Variation de l'éclairement énergétique spectrique en fonction du temps, à trois longueurs d'onde, de la source transportable de contrôle.

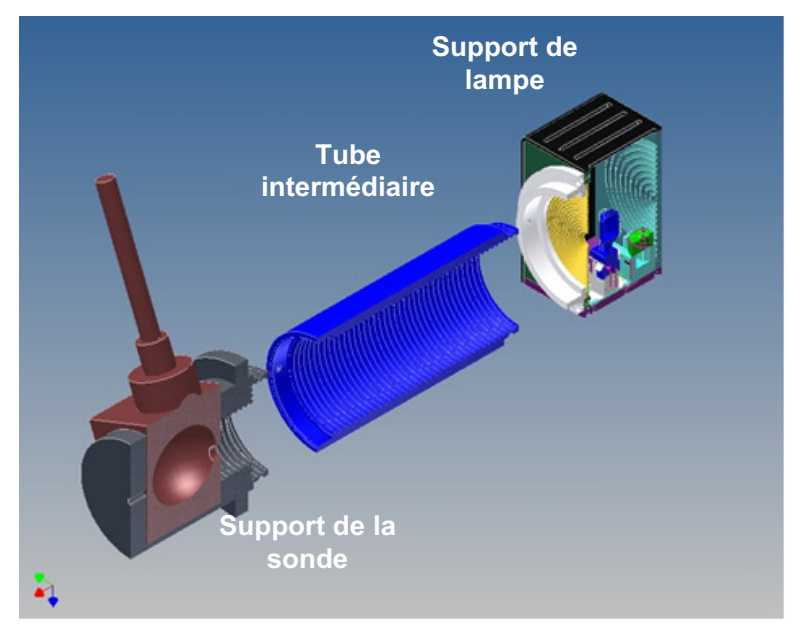

Fig. 10. - Vue éclatée de la source transportable de contrôle.

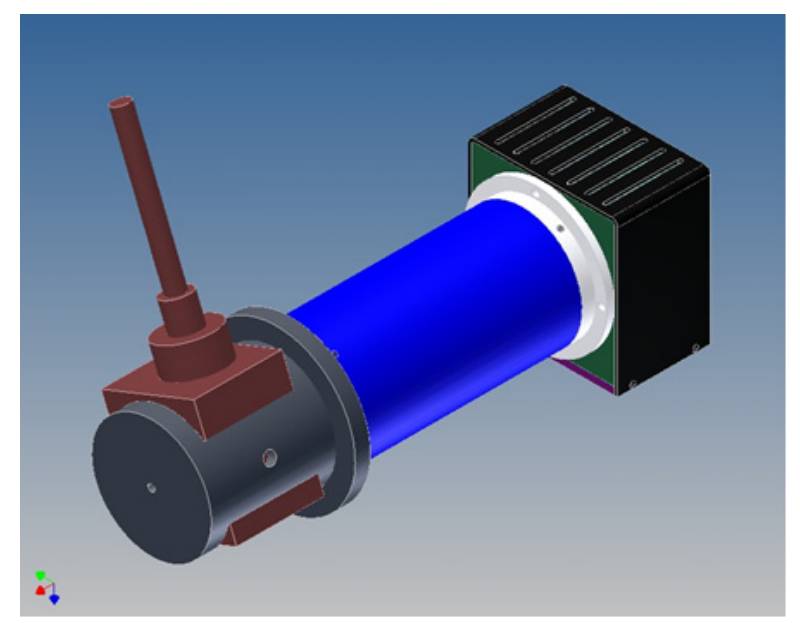

Fig. 11. - Source transportable de contrôle.

est inférieure à $0,1 \%$ sur l'ensemble du domaine spectral. (voir discussion au $§ 5.1$ ).

L'incertitude d'étalonnage en éclairement de la source transportable est indiquée dans le tableau 4.

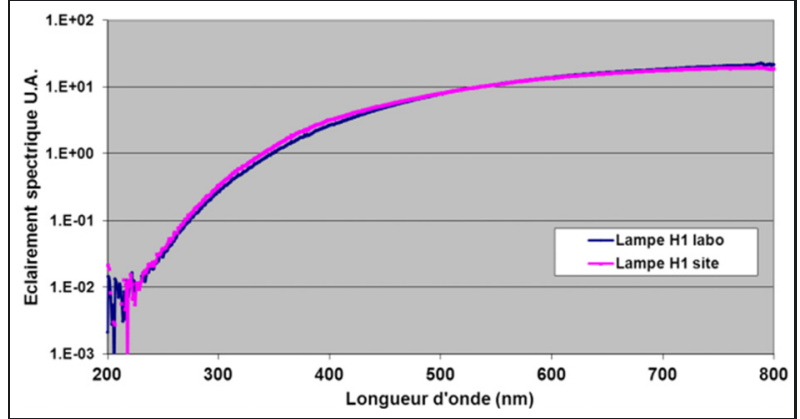

Fig. 12. - Spectres d'émission de la source transportable mesurés au laboratoire et sur site.

Elle varie de $2,8 \%$ à $5,2 \%$ selon la bande spectrale considérée.

\section{Conclusion}

Pour répondre au besoin d'étalonnage des spectroradiomètres à matrice de photodiodes, utilisés pour le contrôle des cabines de bronzage, nous avons développé un banc spécifique qui intègre des sources de lumière dont le spectre d'émission est similaire à celui des lampes des cabines de bronzage. Ce banc permet de prendre en compte la lumière parasite inhérente à ce type de spectroradiomètre qui impacte fortement les mesures d'éclairement dans le domaine UV.

Trois types de sources sont suffisants pour couvrir les besoins de contrôle. L'éclairement spectrique de ces sources est mesuré par le spectroradiomètre à matrice et par un spectroradiomètre de référence à balayage couplé à un double monochromateur. Les résultats de l'étalonnage sont les facteurs correctifs à appliquer à la valeur d'éclairement énergétique effectif pondéré par la courbe d'action de l'érythème sur les domaines UVA et UVB respectivement. L'incertitude relative sur les facteurs correctifs est de $7 \%$ pour les UVA et $10 \%$ pour les UVB. Ces valeurs d'incertitude sont identiques 
Tableau 4

Incertitude d'étalonnage de la source transportable de contrôle.

\begin{tabular}{|l|c|c|c|c|}
\hline \multirow{2}{*}{$\begin{array}{c}\text { Causes } \\
\text { d'incertitude }\end{array}$} & \multicolumn{4}{|c|}{ Incertitude évaluée (en \%) } \\
\cline { 2 - 5 } & $\mathbf{2 5 0} \mathbf{~ n m - 3 0 9} \mathbf{~ n m}$ & $\mathbf{3 1 0} \mathbf{~ m m - 3 1 9} \mathbf{~ m m}$ & $\mathbf{3 2 0} \mathbf{~ n m - 3 4 9} \mathbf{~ m m}$ & $\mathbf{3 5 0} \mathbf{~ n m - 4 0 0 ~} \mathbf{~ m}$ \\
\hline Répétabilité & 2 & 1 & 0,5 & 0,5 \\
\hline $\begin{array}{l}\text { Étalonnage du } \\
\text { spectroradiomètre }\end{array}$ & 1,7 & 2,2 & 1,4 & 1,3 \\
\hline $\begin{array}{c}\text { Courant } \\
\text { d'alimentation }\end{array}$ & 0,1 & 0,1 & 0,1 & 0,1 \\
\hline $\begin{array}{c}\text { Incertitude type } \\
\text { combinée }\end{array}$ & 2,6 & 2,4 & 1,5 & 1,4 \\
\hline $\begin{array}{c}\text { Incertitude } \\
\text { élargie }(\boldsymbol{k}=\mathbf{2})\end{array}$ & $\mathbf{5 , 2}$ & $\mathbf{4 , 9}$ & $\mathbf{3 , 0}$ & $\mathbf{2 , 8}$ \\
\hline
\end{tabular}

pour des mesures sur site grâce à l'utilisation d'une source transportable de contrôle caractérisée au LNE permettant de vérifier le calibrage du spectroradiomètre de référence utilisé.

\section{Références}

[1] CÉSARINI J.-P., « Rayonnement ultraviolet et santé », Radioprotection, 42, 3, 2007, 379-392.

[2] ICNIRP, "On health issues of ultraviolet tanning appliances used for cosmetic purposes", Health Physics 84(1), 2003, 119-127.

[3] Lucas R., McMichael T., Smith W. et Armstrong B., "Solar Ultraviolet Radiation. Global burden of disease from solar ultraviolet radiation", Environmental Burden of Disease Series, No. 13, World Health Organization, 2006.

Article reçu le 10 février 2016, version révisée reçue le 22 juillet 2016.
[4] Décret $\mathrm{N}^{\circ}$ 2013-1261 du 27 décembre 2013 (http://www.legifrance.gouv.fr/eli/decret/2013/12/27/ AFSP1319983D/jo/texte).

[5] Zong Y., Brown S.B., JOHNSON B.C., LYKKE K.R. et OHNO Y, "Simple spectral stray light correction method for array spectroradiometers", Appl. Opt., 45, 2006.

[6] Nevas S., Wübbeler G., Sperling A., Elster C. et Teuber A., "Simultaneous correction of bandpass and stray-light effects in array spectroradiometer data", Metrologia, 49, 2, 2012.

[7] READER J. et CoRLISS C.-H., "Wavelengths and Transition Probabilities for Atoms and Atomic Ions", Part 1, US Department of Commerce - NBS, décembre 1980.

[8] "ILV: International lighting vocabulary", Commission Internationale de l'Eclairage, publication CIE S 017/E, 2011.

[9] « Spectre d'action érythémale de référence et dose érythémale normalisée », Norme NF ISO 17166 (CIE S 007/F), juillet 2000 .

[10] "On limits of exposure to ultraviolet radiation of wavelengths between $180 \mathrm{~nm}$ and $400 \mathrm{~nm}$ (incoherent optical radiation)", ICNIRP guidelines, Health Physics, 87, 2, 2004, 171-186. 